Check for updates

Cite this: Phys. Chem. Chem. Phys., 2019, 21, 22531

Received 14th August 2019, Accepted 24th September 2019

DOI: $10.1039 / c 9 c p 04504 j$

rsc.li/pccp

\section{Rapid carbene formation increases ion diffusivity in an imidazolium acetate ionic liquid confined between polar glass plates}

\begin{abstract}
Andrei Filippov, (D) *ab Oleg N. Antzutkin (D) ac and Faiz Ullah Shah (D)*a
1-Ethyl-3-methyl-imidazolium acetate ([EMIM][OAc]) is one of the most widely used ionic liquids for various applications. This study is focussed on the chemical stability of [EMIM][OAc] on the surfaces of polar glass plates. ${ }^{1} \mathrm{H}$ and ${ }^{13} \mathrm{C}$ NMR spectroscopy and NMR diffusometry of [EMIM][OAC] IL confined between glass plates with a specific surface area $10^{5}-10^{6} \mathrm{~m}^{-1}$ are thoroughly investigated. A rapid and spontaneous reaction took place on the surfaces of glass plates leading to the formation of neutral chemical moieties as evident by the appearance of new signals in the ${ }^{1} \mathrm{H}$ NMR spectra. These new products are assigned as $\mathrm{N}$-heterocyclic carbene (NHC) and acetic acid. These neutral chemical moieties have significantly increased the ion diffusivity by dissociation of the cation and the anion in [EMIM][OAC] IL. The yield and rate of formation of NHC and acetic acid are found to increase with the increasing surface area of polar glass plates and the time of contact between the IL and glass surfaces. Based on NMR spectroscopy, a dissociative reaction mechanism is proposed for the formation of free $\mathrm{NHC}$ in the neat [EMIM][OAC] IL.
\end{abstract}

\section{Introduction}

Ionic liquids (ILs) are typically low temperature melting salts composed of organic or inorganic cations and anions, which remain liquid at a temperature below $100{ }^{\circ} \mathrm{C}$. ILs have unique physicochemical properties such as a high ionic conductivity, low vapour pressures, non-flammability and structural designability. ${ }^{1-4}$ These properties make them appropriate candidates for replacing conventional molecular liquids in many process technologies. ILs are regarded as designer solvents due to their tunability of physical and chemical properties based on the molecular design of the cation and anion structures. ${ }^{4,5}$

1-Ethyl-3-methyl-imidazolium acetate ([EMIM][OAc]) is one of the most commonly used ILs for processing cellulose and chitin fibers and films, ${ }^{6-8}$ aerogels ${ }^{9,10}$ and for biomass pretreatment. $^{11}$ [EMIM] [OAc] is used also as a medium for performing chemical reactions, in particular, for derivatisation of polysaccharides. ${ }^{12}$ Many theoretical and experimental studies of neat and mixtures of [EMIM][OAc] have been performed during the last few years. ${ }^{13-16}$ The nature of the hydrogen bonding ability of such ILs attracts much attention due to their potential applications in dissolution of cellulose. ${ }^{17}$ NMR relaxometry has

\footnotetext{
${ }^{a}$ Chemistry of Interfaces, Luleå University of Technology, SE-97187 Luleå, Sweden. E-mail: andrei.filippov@ltu.se, faiz.ullah@ltu.se

${ }^{b}$ Kazan State Medical University, 420012 Kazan, Russia

${ }^{c}$ Department of Physics, Warwick University, Coventry CV4 7AL, UK
}

demonstrated a discontinuity at a concentration of 0.43 mole fraction of water in [EMIM][OAc], which is attributed to the formation of a hydrogen bonded network. ${ }^{14}$ The local and translational dynamics of [EMIM] [OAc] in neat IL and in mixtures has been studied by $T_{1}$ and $T_{2}$ NMR relaxation techniques and NMR diffusometry. ${ }^{13-15}$ Interestingly, the diffusivity of the cations in imidazolium ILs is often higher than the diffusivity of the anions, even when the size of the anion is much smaller compared to that of the cation. ${ }^{13,15,18}$ This effect has been explained by ionic associations in the ILs stimulated by hydrogen bonding: ion aggregates may be weighted towards anions compared to cations. ${ }^{18}$ The ratio of diffusion coefficients of cations and anions decreases as the concentration of the polar solvent increases in IL-solvent mixtures. ${ }^{13,15}$ Both the diffusivity of ions and the relaxation are coupled with viscosity. ${ }^{13,15}$

Confined ILs ${ }^{19-22}$ have been the research focus for the last few years. The diffusivity of ILs confined in aligned carbon nanotubes, ${ }^{23}$ mesoporous carbon ${ }^{24}$ and silicon ${ }^{21,22}$ increases by a factor of 2-3 or even more compared with the diffusivities of ions in the bulk ILs. Enhanced diffusion of ethylammonium cations has been observed for ethylammonium nitrate confined between polar glass plates. ${ }^{19}$ Recently, the effects of a strong external magnetic field on both the diffusivity and NMR relaxation of confined alkylammonium nitrates have been thoroughly studied. ${ }^{25,26}$

Among the advantages of ionic liquids over molecular liquids are their chemical stability and negligible volatility. 
The structural instability of ILs may limit their use in practical applications and thus may compromise their advantages over molecular liquids. A thorough understanding of the decomposition behaviour of imidazolium based ILs, particularly $[\mathrm{EMIM}][\mathrm{OAc}],{ }^{27}$ is crucial because they are one of the most common commercially available ILs. The possibility of deprotonation of the $\mathrm{C} 2-\mathrm{H}$ group of the $[\mathrm{EMIM}]^{+}$cation by means of a basic counter-ion is known to spontaneously lead to the formation of $\mathrm{N}$-heterocyclic carbene (NHC). ${ }^{28-32}$ It has been suggested that the distillation process of [EMIM][OAc] is driven by the formation of NHCs. ${ }^{28}$ Both theoretical ${ }^{32}$ and spectroscopic ${ }^{29,30}$ studies of carbene formation in the gas phase have been presented. However, the presence of carbene in a liquid has been observed only indirectly, via "trapping" reactions. ${ }^{33,34}$ Evidently the charge screening in the liquid phase stabilises ionic species more readily compared to that in the vapour phase. ${ }^{32}$

Nuclear magnetic resonance is one of the most powerful experimental techniques to monitor the physical and chemical changes in ionic liquid systems in bulk as well as in different confinements. One of the main problems in performing NMR in a confined geometry is the background gradient due to the difference in the magnetic susceptibilities of solid and liquid phases of a sample. ${ }^{35}$ Using flat parallel plates as a confinement has certain advantages from a point of view of NMR measurements, because such a geometry allows an almost complete compensation of the background gradient. ${ }^{19}$ Therefore, undistorted NMR spectra and data on molecular and/or ionic dynamics can be obtained using parallel plates. In this work, we investigated the effect of confinement on the dynamics and stability of the most commonly studied ionic liquid [EMIM][OAc] confined between flat and parallel polar glass plates.

\section{Materials and methods}

\subsection{Sample preparation}

[EMIM][OAc] (97\% purity) was purchased from Sigma-Aldrich and was used without any further purification. The chemical structures of the ionic components of this IL are shown in Fig. 1.

The bulk sample was prepared by placing approximately $300 \mu \mathrm{l}$ of the neat [EMIM][OAc] in a standard $5 \mathrm{~mm}$ glass tube and sealed with a plastic stopper to avoid contact with air. The confined IL samples were prepared with glass plates settled in a stack. ${ }^{19,25,26}$ The plates measuring $14 \times 2.5 \times 0.1 \mathrm{~mm}$ (Thermo Scientific Menzel-Gläser, Menzel GmbH, Germany) were used

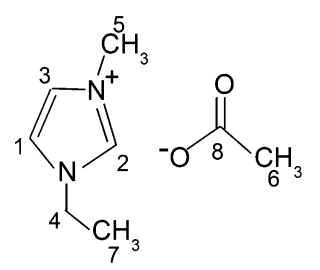

Fig. 1 Chemical structures of the 1-ethyl-3-methyl-imidazolium cation and the acetate anion. Numbers are used to label the protons and carbons in the chemical structures and then implemented in assignments of resonance lines in the NMR spectra. in all the experiments. For one experiment, the plates were thoroughly cleaned using detergent solution and distilled water before sample preparation. These glass plates are referred as "untreated glass plates" in this work. More details on this type of glass plates can be found in our previous publications. ${ }^{19,25,26}$ For another experiment, the glass plates were placed in $4 \mathrm{wt} \%$ hydrofluoric acid (HF) aqueous solution for 40 minutes. After that, the $\mathrm{HF}$ treated glass plates were cleaned in the same manner as the untreated plates and referred to as "HF treated glass plates" in this work. A stack of glass plates filled with neat [EMIM][OAc] was prepared in a glove box under a dry $\mathrm{N}_{2}$ atmosphere. The samples were prepared by dropping $2 \mu \mathrm{L}$ of the IL onto the first glass plate, placing another glass plate on the top, adding $2 \mu \mathrm{L}$ of the IL on the top of this glass plate, etc. This process was continued until the thickness of the stack reached $2.5 \mathrm{~mm}$. The $\mathrm{IL}$ that overflowed at the edges of the stack was removed by sponging. The sample consisting of a stack of $c a .37$ glass plates was positioned in a rectangular glass tube and sealed. The mean spacing between the untreated glass plates was assessed by weighing the introduced IL, which yielded $d \sim 3.8-4.5 \mu \mathrm{m} .{ }^{19}$ A detailed description of the sample preparation and characterisation has been reported in our previous reports. ${ }^{19,25,26,36}$ Just before measurements, each sample was vacuum treated at pressures less than $10^{-3}$ bar for 12 hours to remove volatile impurities. All measurements were performed at $294 \mathrm{~K}$.

\subsection{NMR spectroscopy}

NMR experiments were performed on a Bruker Ascend/Aeon WB 400 (Bruker BioSpin AG, Fällanden, Switzerland) NMR spectrometer. The working frequencies for ${ }^{1} \mathrm{H}$ and ${ }^{13} \mathrm{C}$ NMR were 400.21 and $100.64 \mathrm{MHz}$, respectively. The NMR spectra were recorded using a simple single $\pi / 2$ radiofrequency pulse. The $\pi / 2$ pulse durations were $7 \mu$ s and $6 \mu$ s for ${ }^{1} \mathrm{H}$ and ${ }^{13} \mathrm{C}$, respectively. All resonance lines in the ${ }^{1} \mathrm{H}$ and ${ }^{13} \mathrm{C}$ NMR spectra were externally referenced to tetramethylsilane (TMS).

\section{3. ${ }^{1} \mathrm{H}$ NMR diffusometry}

${ }^{1} \mathrm{H}$ NMR self-diffusion measurements of the IL were performed with a Pulsed-Field-Gradient (PFG) probe Diff50 (Bruker). The diffusional decays (DD) were recorded using a spin-echo pulse sequence. For single-component isotropic diffusion, the signal intensity, $A$, changes as: ${ }^{37,38}$

$$
A(\tau, g, \delta) \propto \exp \left(-\frac{2 \tau}{T_{2}}\right) \exp \left(-\gamma^{2} \delta^{2} g^{2} D t_{\mathrm{d}}\right)
$$

Here, $\tau$ is the time interval in the pulse sequence; $\gamma$ is the gyromagnetic ratio for nuclei; $g$ and $\delta$ are the amplitude and the duration of the gradient pulse; $t_{\mathrm{d}}=(\Delta-\delta / 3)$ is the diffusion time; and $\Delta$ is the time interval between gradient pulses. $D$ is the diffusion coefficient. Typical experimental conditions included signal averaging with 32 scans, 32 gradient steps, a total gradient pulse length of $\delta=1.5 \mathrm{~ms}$, and a $5 \mathrm{~s}$ recycle delay. $\Delta$ was $20 \mathrm{~ms}$. The amplitude of $g$ varied from 0.06 up to $15 \mathrm{~T} \mathrm{~m}^{-1}$. 


\section{Results and discussion}

\subsection{Experimental data}

The ${ }^{1} \mathrm{H}$ NMR spectra of the bulk and confined [EMIM][OAc] obtained after regular intervals over a few days are shown in Fig. 2. The ${ }^{1} \mathrm{H}$ NMR spectrum of the bulk [EMIM][OAc] sample coincides with the previously recorded spectra by other researchers. ${ }^{13,15}$ The resonance line assignment is made according to the published reports, ${ }^{15}$ as shown in Fig. 2a. No significant changes are observed in the ${ }^{1} \mathrm{H}$ NMR spectrum of the bulk [EMIM][OAc] sample during three months after keeping the NMR sample tube at room temperature. This means that the IL is stable under these conditions in accordance with a previous study. ${ }^{27}$ For [EMIM][OAc] confined between glass plates (Fig. 2b-g), significant changes are noticed in the ${ }^{1} \mathrm{H}$ NMR spectra already after 1.5 hours when placed between the glass plates (Fig. 2c). A new ${ }^{1} \mathrm{H}$ resonance line appeared at $c a$. 5.9 ppm after 1.5 hours and grew in intensity with time. The intensity of this line was gradually increased and a "saturation point" was reached after two days of placing the IL between the glass plates (Fig. 2g). At the same time the resonance lines 1, 2 and 3 gradually shifted towards lower ppm values. In addition to the change in the chemical shift, the resonance lines assigned to the 1-3 protons became broader during the first 6 hours. This change in the chemical shifts and line broadening are typical for a proton chemical exchange in the intermediate exchange regime. ${ }^{39}$ Therefore, in the beginning of the experiment (up to $\sim 4 \mathrm{~h}$ ) the system experienced a transition from a slow exchange to an intermediate exchange regime, while at a longer time, a new transition from an intermediate to a slow exchange regime occurred. For the observed coalescence, we can calculate the exchange rate using the following equation: ${ }^{39}$

$$
K_{\mathrm{c}}=\pi \Delta \nu / \sqrt{2}
$$

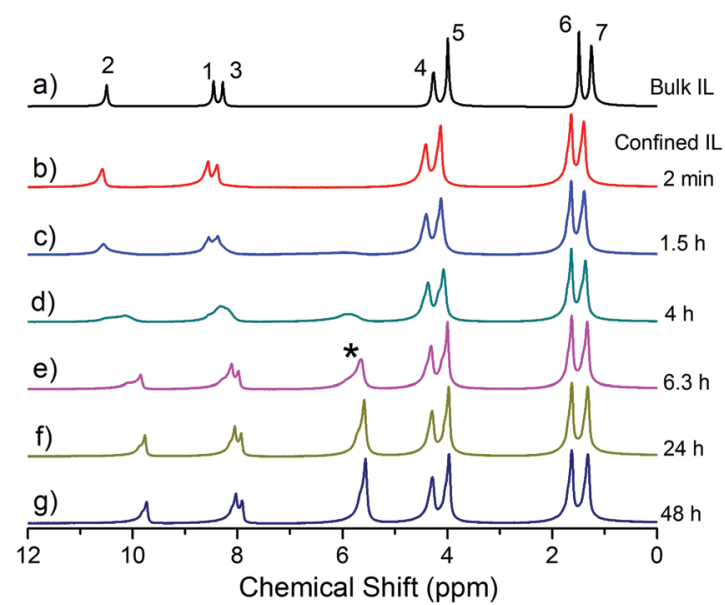

Fig. $2{ }^{1} \mathrm{H}$ NMR spectra of [EMIM][OAc] in the bulk (a) and confined between $\mathrm{HF}$ treated glass plates recorded at regular intervals of time $(b-g)$. The resonance lines are assigned to the chemical groups of [EMIM][OAc] according to the previously published work. ${ }^{15} \mathrm{~A}$ new resonance line at 5.9 ppm appeared in the spectrum which is marked by an asterisk. where $\Delta \nu$ is the difference of the chemical shifts in frequency units of the two exchanging spin sites. For resonance lines 2 and 3 the difference in their frequencies is $70.1 \mathrm{~Hz}$, and therefore, $K_{\mathrm{c}}$ is $155.6 \mathrm{~s}^{-1}$ as calculated using eqn (2).

The ${ }^{13} \mathrm{C}$ NMR spectra of [EMIM][OAc] in the bulk and confined between $\mathrm{HF}$ treated glass plates after two days are shown in Fig. 3. The ${ }^{13} \mathrm{C}$ resonance lines are assigned using a "Predict ${ }^{13} \mathrm{C}$ carbon NMR spectra software". ${ }^{40}$ The spectra of the bulk and confined [EMIM][OAc] are coincident except for the resonance line of carbons 2 and 8 . The doublet for carbon 2 is slightly shifted towards lower ppm values from $140.4 \mathrm{ppm}$ to $138.8 \mathrm{ppm}$ and the singlet for the carboxylate carbon 8 is shifted toward higher chemical shift values from $175.9 \mathrm{ppm}$ to $177.5 \mathrm{ppm}$. This clearly indicates that chemical changes occurred in the structure of the imidazolium cation of the IL at the glass surfaces. In general, ${ }^{13} \mathrm{C}$ NMR spectroscopy appeared to be less sensitive to such structural changes that occurred with the confined [EMIM][OAc] compared to ${ }^{1} \mathrm{H}$ NMR spectroscopy (Fig. 2).

After recording the NMR spectra of [EMIM][OAc] confined between glass plates for two days, the sample was again vacuum treated for 12 hours at $10^{-3}$ mbar pressure and room temperature. The resonance line at $\sim 5.9 \mathrm{ppm}$ disappeared from the ${ }^{1} \mathrm{H}$ NMR spectrum of the freshly vacuum treated sample and the spectrum looked like the one shown in Fig. 2b. This reveals that the new products formed on the surfaces of the glass plates are volatile and have left the system under vacuum for 12 hours. To confirm the repeatability of this process, the same was repeated and the ${ }^{1} \mathrm{H}$ NMR spectra were recorded at regular intervals. It was found that the process shown in Fig. $2 \mathrm{~b}-\mathrm{g}$ with the appearance of the new resonance line at $\sim 5.9 \mathrm{ppm}$ and increasing intensity up to saturation was reproducible. This procedure was then repeated several times, and the spectral changes as a function of time were reproduced each time. Therefore, confined [EMIM][OAc] degrades producing volatile products with a new resonance line at $\sim 5.9 \mathrm{ppm}$ in the ${ }^{1} \mathrm{H}$ NMR spectra. The volatile products can be pumped out by vacuum treatment to obtain the neat [EMIM][OAc] again.

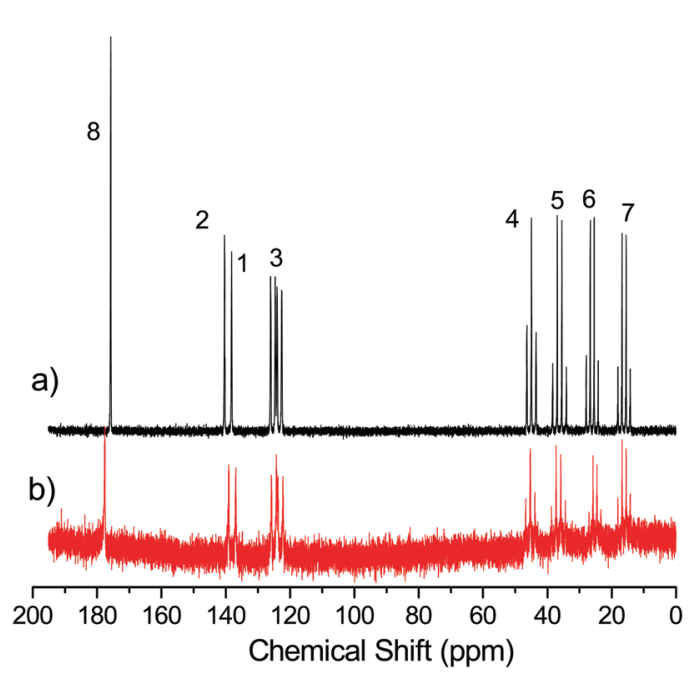

Fig. $3{ }^{13} \mathrm{C}$ NMR spectra of [EMIM][OAc] in the bulk (a) and confined between HF treated glass plates after two days (b). 
Generally, a change in the molecular structure leads to a change in the dynamic properties of the molecules or ions in a liquid. The change in the dynamic properties can be monitored by measuring the diffusivity of molecules and ions. Therefore, we measured the diffusion coefficients of [EMIM][OAc] in bulk and in confinement between the glass plates at regular intervals for a few days. The diffusion of the $[\mathrm{EMIM}]^{+}$cation and the $[\mathrm{OAc}]^{-}$anion was measured using diffusion decays (eqn (1)) of the corresponding resonance lines of the ${ }^{1} \mathrm{H}$ NMR spectra (lines 1-5 and 7 for the imidazolium cation and line 6 for the acetate anion). The diffusion coefficient of a species corresponding to a new resonance line that appeared in the ${ }^{1} \mathrm{H}$ NMR spectrum of the confined [EMIM][OAc] at $\sim 5.9$ ppm (Fig. 2, asterisk) was also measured in the experiment. The bulk [EMIM][OAc] values of the diffusion coefficient for cations and anions at $294 \mathrm{~K}$ are $6.9 \times 10^{-12} \mathrm{~m}^{2} \mathrm{~s}^{-1}$ and $5.7 \times 10^{-12} \mathrm{~m}^{2} \mathrm{~s}^{-1}$, respectively. These values are comparable to the previously reported diffusion coefficients for the neat bulk [EMIM][OAc] ionic liquid. ${ }^{13,15}$ It is observed that the diffusion coefficient of the acetate anion is smaller than that of the imidazolium cation, which is in accordance with the previous reports on [EMIM][OAc] and other imidazolium ILs. ${ }^{13,15,18}$ Noteworthily, for a bulk sample of [EMIM][OAc] neither new resonance lines appeared, nor the values of diffusion coefficients for the imidazolium cation and the acetate anion changed during three months of the sample placement in the NMR tube. This suggests that no change has occurred in the structure of the bulk [EMIM][OAc] IL in the NMR tube. In contrast, significant changes occurred in the structures of the [EMIM][OAc] IL that led to an increase in the diffusion coefficients of cations, anions and the volatile components, when the [EMIM][OAc] IL was confined between glass plates. Dependences of diffusion coefficients on the time of the experiment, as well as the intensity of the new resonance line at $\sim 5.9 \mathrm{ppm}$ corresponding to a volatile product of the IL decomposition, are shown in Fig. 4. As is clearly seen in Fig. 4, the diffusivities of the imidazolium cation, the acetate anion and the new product increase in a concerted way as a function of time. In addition, the intensity of the resonance line corresponding to the new product also increases in a similar fashion to the diffusivities of the components.

A pertinent question is what is the reason for the increase in the diffusion coefficients of [EMIM][OAc] between the HF treated glass plates? It was observed earlier that an increase in the amount of a molecular solvent or a molecular impurity lead to an increase in the diffusion coefficients of ions. ${ }^{19,41,42}$ This is mainly due to the dissociation of ions by the solvent, which leads to a decrease in the inter-ionic interactions and viscosity of the ionic liquid.

In our previous studies, we observed a change in the diffusion coefficients and NMR relaxation times of alkylammonium nitrate ILs confined between polar glass plates and placed in an external static magnetic field of $9.4 \mathrm{~T}$ as a function of timeexposure in the magnetic field. ${ }^{25,26,36}$ Therefore, we investigated the effect of an external static magnetic field on the ${ }^{1} \mathrm{H}$ NMR spectra and diffusivities of [EMIM][OAc]. For this, in a separate experiment the confined [EMIM][OAc] IL between glass plates

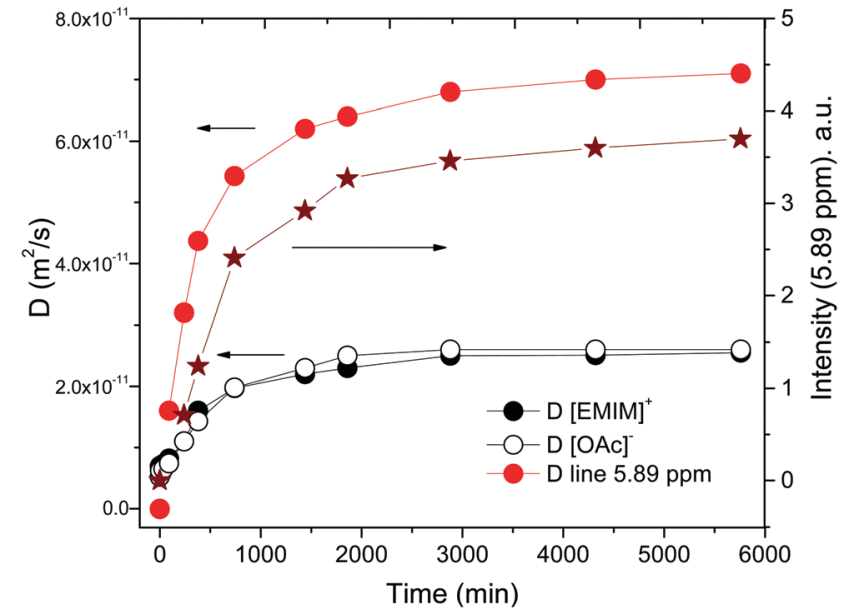

Fig. 4 Variation of the diffusion coefficients of the imidazolium cation (filled black circles), the acetate anion (empty black circles) and the new resonance line at $5.9 \mathrm{ppm}$ (filled red circles) of [EMIM][OAc] confined between $\mathrm{HF}$ treated glass plates. An increase in the integral intensity of the new resonance line at $5.9 \mathrm{ppm}$ (stars) in the ${ }^{1} \mathrm{H}$ NMR spectrum of the confined [EMIM][OAc] between HF treated glass plates. Arrows point towards the $Y$-axis for each curve.

was kept outside the magnetic field of the NMR spectrometer most of the time and was placed in the spectrometer only for a short time $(\sim 3 \mathrm{~min})$ of measurements. The same effect for the ${ }^{1} \mathrm{H}$ NMR spectrum and the diffusivities of ions was observed for the sample which was placed inside the NMR probe throughout the experiment. Therefore, the effect of the static magnetic field, if it exists in this system, is negligibly smaller compared to the effect of the confinement between polar glass plates.

\subsection{N-heterocyclic carbene formation mechanism}

Although the thermal decomposition temperature of [EMIM][OAc] IL is higher than $200{ }^{\circ} \mathrm{C}$, it is known to be deprotonated by the acetate anion. ${ }^{27}$ The deprotonation leads to formation of $\mathrm{N}$-heterocyclic carbene (NHC) which is a very efficient ligand and organocatalyst. Therefore, great interest has been seen over the past decades in this particular ionic liquid. The mechanism of NHC formation and its presence in [EMIM][OAc] IL is still debated in the scientific community. On the one side, the transformation of charged species into neutral molecules via proton transfer from the $\mathrm{C} 2-\mathrm{H}$ group of the imidazolium cation to the acetate anion is unfavourable due to the highly polar environment of this system. ${ }^{29}$ On the other side, it has been experimentally demonstrated that imidazolium acetate based ILs can react as carbene with metal ions, ${ }^{43}$ metal oxides, ${ }^{44}$ carbon dioxide ${ }^{45}$ and chalcogens ${ }^{46}$ forming carbene-adducts or complexes with these substrates.

There are two plausible reaction mechanisms of carbene formation in [EMIM][OAc] IL, the first one is a dissociative reaction mechanism and the second one is an associative reaction mechanism. In the dissociative mechanism, proton transfer occurs from the $\mathrm{C} 2-\mathrm{H}$ group of the imidazolium cation to the basic acetate anion leading to the formation of free carbene and acetic acid in the system. The highly reactive carbene moiety 
then reacts with the substrate forming a covalent bond. On the other hand, the proton transfer and the chemical bond formation take place through a concerted reaction path. Recently, Gehrke et $a l .{ }^{47}$ have performed electrochemical studies on neat [EMIM] [OAc] IL and found that the associative reaction mechanism is slightly more favourable on the surface of platinum, which was further supported by density functional theory (DFT) calculations and $a b$ initio molecular dynamics (AIMD) simulations. These authors have suggested that the mechanism of reaction is determined by the reaction conditions, the type of substrate and the side chain of the imidazolium cation. Therefore, it is not possible to draw a general conclusion on the reaction mechanism of carbene formation.

We suggest that the carbene formation in [EMIM][OAc] IL confined between glass plates is a result of the catalytic activity of the glass surface. To further confirm the effect of the surface, we studied [EMIM][OAc] IL confined between two types of glass plates, i.e. HF treated glass plates and untreated glass plates. NMR diffusometry was employed to investigate the influence of the substrate on the ion diffusion in [EMIM][OAc] IL. The effect of the substrate on the ion diffusivities by the surfaces of the untreated glass plates and the HF treated glass plates is shown in Fig. 5. It is clearly seen that the ion diffusivity in [EMIM][OAc] IL increases as a function of time in the case of both the untreated glass plates and the HF treated glass plates. However, both the rate of change of $D$ and the level of saturation are much higher in the case of [EMIM][OAc] IL on the HF treated glass plates.

Now we can relate the observed effects with the specific areas of the studied systems. The specific area in the case of a liquid placed in a $5 \mathrm{~mm}$ outer diameter NMR tube is $\sim 10^{3} \mathrm{~m}^{-1}$. The specific surface area of the untreated glass plates (estimated from the mean distance between plates $\sim 3.8 \mu \mathrm{m})^{36}$ is $\sim 5.3 \times 10^{5} \mathrm{~m}^{-1}$. However, the specific surface area of the glass

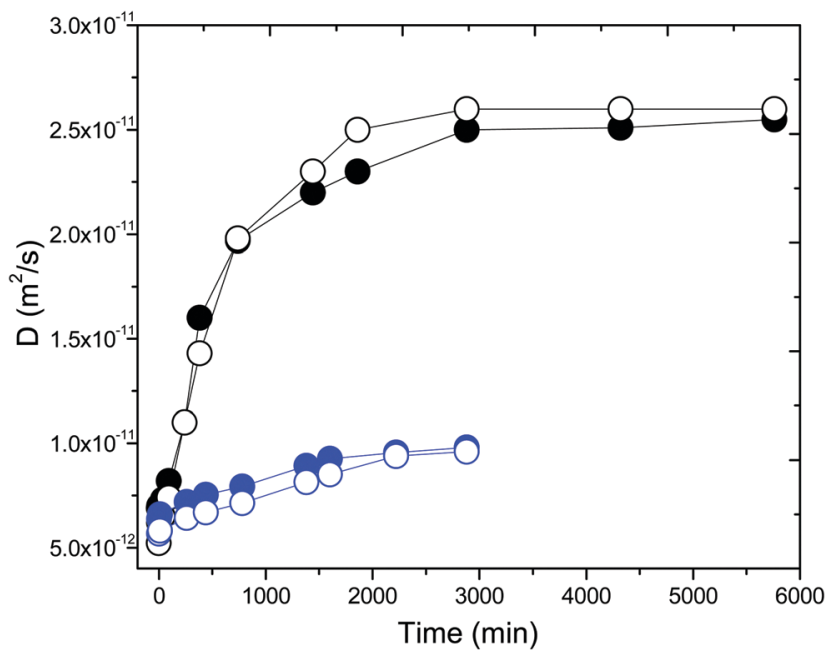

Fig. 5 Variation of diffusion coefficients of cations (solid circles) and anions (open circles) of [EMIM][OAc] IL confined between the untreated glass plates (blue circles) and the HF treated glass plates (black circles, from Fig. 4). plates changes when the surface is HF etched. When a smooth surface is etched, the surface roughens and cusp-like structures are formed. ${ }^{48}$ This formation is related to the presence of flaws in the surface before etching. The size or depth of these flaws changes from microcracks to larger cracks after polishing, which are formed as a result of mechanical actions such as grinding or particle erosion. The closed flaws or microcracks are etched open and this surface defect is gradually transformed into a cusp as etching proceeds. ${ }^{48}$ The untreated and HF treated glass plates were analysed by the BrunauerEmmett-Teller (BET) method, which is usually used to quantify the specific surface area. ${ }^{36}$ Nitrogen adsorption-desorption data were recorded at $77 \mathrm{~K}$ on a Micromeritics ASAP 2010 instrument. Prior to measurements, the samples were outgassed at $300 \mathrm{~K}$ under vacuum for $10 \mathrm{~h}$. The BET surface areas were $0.1 \pm 0.01 \mathrm{~m}^{2} \mathrm{~g}^{-1}$ and $0.05 \pm 0.01 \mathrm{~m}^{2} \mathrm{~g}^{-1}$ for the untreated and $\mathrm{HF}$ etched glass plates, respectively. These surface areas were contributed mainly by microcracks (micropores with sizes less than $2 \mathrm{~nm}$ ), in which penetration of [EMIM][OAc] IL was difficult or even impossible. At the same time, the volume of the microcracks was extremely small, $\sim 0.000011 \mathrm{~cm}^{3} \mathrm{~g}^{-1} \cdot{ }^{36}$ Therefore, the specific surface area of the HF treated glass plates is $\sim 9.5 \times 10^{5} \mathrm{~m}^{-1}$. This reveals that an increase in the roughness of the plate surfaces leads to an increase in the specific surface area by a factor of $\sim 1.8$. Therefore, the use of glass plates increases the specific surface area of the glass by a factor of hundred in comparison with the standard NMR tube. The specific surface area on which the process of carbene formation in [EMIM] [OAc] IL occurs is of the order $10^{5}-10^{6} \mathrm{~m}^{-1}$. The rate and completeness of carbene formation in [EMIM][OAc] IL is directly correlated with the specific surface area of the contact surfaces. This means that the higher the specific surface area of the glass plates, the larger the amount of carbene formation.

Taking into account the obtained NMR spectra and kinetic dependences, we can suggest that the mechanism of chemical reactions occurring in [EMIM] [OAc] IL in the presence of glass surfaces is through a dissociative path. Firstly, the products of the reaction are electrically neutral and volatile, because the new products were easily removed from the IL when placed under vacuum. Secondly, the product of dissociation exchanges with $2,1,3$ protons of the [EMIM] $]^{+}$cation as confirmed by the ${ }^{1} \mathrm{H}$ NMR spectra. Based on the NMR analysis, the proposed chemical reaction is shown in Fig. 6. This reaction is a typical deprotonation of the $\mathrm{C} 2-\mathrm{H}$ group of the $[\mathrm{EMIM}]^{+}$cation by a basic counter-ion leading to the spontaneous formation of NHCs, as observed previously. ${ }^{28-32}$ Therefore, the products of dissociation of [EMIM][OAc] IL on the glass surfaces are $\mathrm{N}$-heterocyclic carbene (NHC) and acetic acid.

Indeed, the chemical shifts of protons 1 and 3 in NHC, as shown by simulation with a NMR software, ${ }^{40}$ are at $6.05 \mathrm{ppm}$. The proton chemical shifts for the $\mathrm{CH}_{2}$ and $\mathrm{CH}_{3}$ groups are shifted to lower ppm values relative to the $[\mathrm{EMIM}]^{+}$cation, while the chemical shifts for the protons in acetic acid remained at the same position as for the $[\mathrm{OAc}]^{-}$anion. The reaction shown in Fig. 6 has been suggested earlier during distillation of [EMIM][OAc] $\mathrm{IL}^{28}$ but its products NHC and acetic acid have not been directly observed 


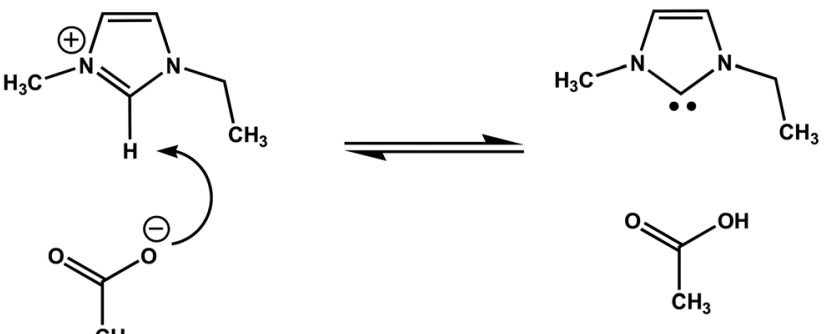

Fig. 6 Suggested dissociative reaction mechanism of carbene formation in [EMIM][OAc] IL confined between glass plates.

in the liquid phase. ${ }^{29,32}$ This suggests that confinement of [EMIM][OAc] IL between polar glass plates stabilises the dissociation products. The bulk [EMIM][OAc] IL smells slightly as acetic acid. This means that traces of acetic acid are present in the bulk IL, but the equilibrium is shifted far left, in accordance with the previously reported data. ${ }^{29,32}$ The confined IL and the dissociation products are in a quasi-equilibrium state as confirmed by the changes in the ${ }^{1} \mathrm{H}$ NMR spectra (Fig. 2) on the surfaces of glass plates. The presence of the glass surface shifted this equilibrium to the right. Due to kinetic reasons, this process takes place for two days. In the middle of the NHC formation process, there is interconversion between [EMIM $]^{+}$ and NHC, which is observed in the NMR spectra as a "proton exchange" in the intermediate exchange regime that leads to ${ }^{1} \mathrm{H}$ NMR resonance line broadening for the protons $1,2,3$ (Fig. 2b-e). We believe that the chemical exchange is retarded at low (beginning of the experiment) and high (at the end of experiment) concentrations of NHC. After the observed intermediate exchange and lines coalescence (Fig. 2d), no fast exchange occurs, evidently because of the quite low mobilities of [EMIM $]^{+}$ and NHC at room temperature. Therefore, the suggested dissociation reaction presented in Fig. 6 is in agreement with the changes in the ${ }^{1} \mathrm{H}$ and ${ }^{13} \mathrm{C}$ NMR spectra (Fig. 2 and 3). The neutral NHC and acetic acid act as solvents in the IL. They do not interact electrostatically and thus the viscosity of the system decreases that leads to an increase in the diffusivity of all the ionic and molecular species present in the system.

\section{Conclusions}

${ }^{1} \mathrm{H}$ and ${ }^{13} \mathrm{C}$ NMR spectroscopy and ${ }^{1} \mathrm{H}$ NMR diffusometry were performed on the [EMIM][OAc] ionic liquid confined between glass plates with a specific surface area of $10^{5}-10^{6} \mathrm{~m}^{-1}$. The presence and an increase with time in the fraction of neutral and volatile products of dissociation of the [EMIM] [OAc] IL were detected by both, ${ }^{1} \mathrm{H}$ NMR and ${ }^{13} \mathrm{C}$ NMR spectroscopy and diffusometry. In contrast, no such dissociation products were observed when the [EMIM][OAc] IL was placed in an NMR tube for the same period of time. The rate and degree of dissociation increased as the specific surface area of the glass plates increased. Based on the NMR spectroscopy data, the products of the dissociation reaction were found to be N-heterocyclic carbene and acetic acid and the plausible mechanism was a dissociative path where free carbene was present in the liquid.
The effect of interconversion between the $[\mathrm{EMIM}]^{+}$cation and $\mathrm{N}$-heterocyclic carbene was observed in the ${ }^{1} \mathrm{H}$ NMR spectra as a "proton exchange" in the slow-intermediate exchange regime. Dissociation reactions in the confined [EMIM][OAc] IL and the potential formation of $\mathrm{N}$-heterocyclic carbenes require more detailed studies because of the high level of practical interest in various applications.

\section{Conflicts of interest}

The authors declare no conflict of interest.

\section{Acknowledgements}

The financial support from the Norrbotten Research Council (NoFo 19-100) and the Swedish Research Council (project number: 2018-04133) is acknowledged for this work.

\section{References}

1 T. Welton, Room-temperature ionic liquids: solvents for synthesis and catalysis, Chem. Rev., 1999, 99, 2071-2083.

2 P. Wassersheid and W. Keim, Ionic liquids - new "solutions" for transition metal catalysis, Angew. Chem., Int. Ed., 2000, 39, 3772-3789.

3 L. A. Aslanov, Ionic liquids: liquid structure, J. Mol. Liq., 2011, 162, 101-104.

4 N. V. Plechkova and K. R. Seddon, Applications of ionic liquids in the chemical industry, Chem. Soc. Rev., 2008, 37, 123-150.

5 S. Bhattacharyya, A. Filippov and F. U. Shah, Insight into the effect of $\mathrm{CO}_{2}$ absorption on the ionic mobility of ionic liquids, Phys. Chem. Chem. Phys., 2016, 18, 28617-28652.

6 B. Kosan, C. Michels and F. Meister, Dissolution and forming of cellulose with ionic liquids, Cellulose, 2008, 15, 59-66.

7 T. Cai, H. Zhang, Q. Guo, H. Shao and X. Hu, Structure and properties of cellulose fibers from ionic liquids, J. Appl. Polym. Sci., 2009, 115, 1047-1053.

8 Y. Qin, X. Lu, N. Sun and R. D. Rogers, Dissolution or extraction of crustacean shells using ionic liquids to obtain high molecular weight purified chitin and direct production of chitin films and fibers, Green Chem., 2010, 12, 968-971.

9 O. Aaltonen and O. Jauhiainen, The preparation of lignocellulosic aerogels from ionic liquid solutions, Carbohydr. Polym., 2009, 75, 125-129.

10 R. Sescousse, R. Gavillon and T. Budtova, Aerocellulose from cellulose-ionic liquid solutions: preparation, properties and comparison with cellulose-NaOH and cellulose-NMMO routes, Carbohydr. Polym., 2011, 83, 1766-1774.

11 A. Brandt, J. Gräsvik, J. P. Hallett and T. Welton, Deconstruction of lignocellulosic biomass with ionic liquids, Green Chem., 2013, 15, 550-583.

12 T. Heinze, K. Schwikal and S. Barthel, Ionic liquids as reaction medium in cellulose functionalization, Macromol. Biosci., 2005, 5, 520-525. 
13 S. M. Green, M. E. Ries, J. Moffat and T. Budtova, NMR and rheological study of anion size influence on the properties of two imidazolium-based ionic liquids, Sci. Rep., 2017, 7, 8968.

14 J. J. Allen, S. R. Bowser and K. Damodaran, Molecular interactions in the ionic liquid emim acetate and water binary mixtures probed via NMR spin relaxation and exchange spectroscopy, Phys. Chem. Chem. Phys., 2014, 16, 8078-8085.

15 A. Radhi, K. A. Le, M. E. Ries and T. Budtova, Macroscopic and microscopic study of 1-ethyl-3-methyl-imidazolium acetateDMSO mixtures, J. Phys. Chem. B, 2015, 119, 1633-1640.

16 M. Brehm, H. Weber, A. S. Pensado, A. Stark and B. Kirchner, Proton transfer and polarity changes in ionic liquid-water mixtures: a perspective on hydrogen bonds from ab initio molecular dynamics at the example of 1ethyl-3-methylimidazolium acetate-water mixtures - part 1, Phys. Chem. Chem. Phys., 2014, 14, 5030-5044.

17 Z. Papanyan, C. Roth, D. Paschek and R. Ludwig, Understanding the dissolution of polyols by ionic liquids using the example of a well-defined model compound, ChemPhysChem, 2011, 12, 2400-2404.

18 H. Tokuda, K. Hayamizu, K. Ishii, A. B. H. Susan and M. Watanabe, Physicochemical properties and structures of room temperature ionic liquids. 1. Variation of anionic species, J. Phys. Chem. B, 2004, 108, 16593-16600.

19 A. Filippov, O. I. Gnezdilov, N. Hjalmarsson, O. N. Antzutkin, S. Glavatskih, I. Furó and M. W. Rutland, Acceleration of diffusion in ethylammonium nitrate ionic liquid confined between parallel glass plates, Phys. Chem. Chem. Phys., 2017, 19, 25853-25858.

20 S. Zhang, J. Zhang, Y. Zhang and Y. Deng, Nanoconfined ionic liquids, Chem. Rev., 2017, 117, 6755-6833.

21 C. Iacob, J. R. Sangoro, P. Papadopoulus, T. Schubert, S. Naumov, R. Valiullin, J. Kärger and F. Kremer, Charge transport and diffusion of ionic liquids in nanoporous silica membranes, Phys. Chem. Chem. Phys., 2010, 12, 13798-13803.

22 A. Filippov, N. Azancheev, F. U. Shah, S. Glavatskih and O. N. Antzutkin, Self-diffusion of phosphonium bis(salicylato)borate ionic liquid in pores of Vycor porous glass, Microporous Mesoporous Mater., 2016, 230, 128-134.

23 Q. Berrod, F. Ferdeghini, P. Judenstein, N. Genevaz, R. Ramos, A. Fournier, J. Dijon, J. Ollivier, S. Rols, D. Yu, R. A. Mole and J.-M. Zanotti, Enhanced ionic liquid mobility induced by confinement in 1D CNT membranes, Nanoscale, 2016, 8, 7845-7848.

24 S. M. Chathoth, E. Mamontov, P. F. Fulvio, X. Wang, G. A. Baker, S. Dai and D. J. Wesolowski, An unusual slowdown of fast diffusion in a room temperature ionic liquid confined in mesoporous carbon, Europhys. Lett., 2013, 102, 16004.

25 A. Filippov and O. N. Antzutkin, Magnetic field effects dynamics of ethylammonium nitrate ionic liquid confined between glass plates, Phys. Chem. Chem. Phys., 2018, 20, 6316-6320.

26 A. Filippov, O. I. Gnezdilov and O. N. Antzutkin, Static magnetic field alters properties of confined alkyammonium nitrate ionic liquids, J. Mol. Liq., 2018, 268, 49-54.

27 M. T. Clough, K. Geyer, P. A. Hunt, J. Mertes and T. Welton, Thermal decomposition of carboxylate ionic liquids: trends and mechanisms, Phys. Chem. Chem. Phys., 2013, 15, 20480-20495.

28 M. J. Earle, J. M. S. S. Esperanca, M. A. Gilea, J. N. Canongia Lopes, L. P. N. Rebelo, J. W. Magee, K. R. Seddon and J. A. Widergren, The distillation and volatility of ionic liquids, Nature, 2006, 439, 831-834.

29 I. Chiarotto, L. Mattiello, F. Pandolfi, D. Rocco and M. Feroci, NHC in imidazolium acetate ionic liquids: actual or potential presence? Front. Chem., 2018, 6, 355.

30 J. R. Diniz, T. B. de Lima, R. Galaverna, A. L. de Oliveira, D. A. C. Ferreira, F. C. Gozzo, M. N. Eberlin, J. Dupont and B. A. D. Netto, Is the formation of N-heterocyclic carbines (NHCs) a feasible mechanism for the distillation of imidazolium ionic liquids? Phys. Chem. Chem. Phys., 2018, 20, 24716-24725.

31 N. M. A. N. Daud, E. Bakis, J. P. Hallett, C. C. Weber and T. Welton, Evidence for the spontaneous formation of $\mathrm{N}$-heterocyclic carbenes in imidazolium based ionic liquids, Chem. Commun., 2017, 53, 11154-11156.

32 O. Holloczki, D. S. Firaha, J. Friedrich, M. Brehm, R. Cybic, M. Wild, A. Stark and B. Kirchner, Carbene formation in ionic liquids: spontaneous, induced, or prohibited? J. Phys. Chem. B, 2013, 117, 5898-5907.

33 G. Gurau, H. Rodriguez, S. P. Kelley, P. Janiczek, R. S. Kalb and R. D. Rogers, Demonstration of chemosorption of carbon dioxide in 1,3-dialkylimidazolium acetate ionic liquids, Angew. Chem., Int. Ed., 2011, 50, 12024-12026.

34 Z. Klemen, O. Holloczki, J. Nagy and L. Nyulaszi, An organocatalytic ionic liquid, Org. Biomol. Chem., 2011, 9, 5362-5364.

$35 \mathrm{~J}$. A. Glasel and K. H. Lee, On the interpretation of water nuclear magnetic resonance relaxation times in heterogeneous systems, J. Am. Chem. Soc., 1974, 96, 970-978.

36 A. Filippov, O. I. Gnezdilov, A. G. Luchkin and O. N. Antzutkin, Self-diffusion of ethylammonium nitrate ionic liquid confined between modified polar glasses, J. Mol. Liq., 2019, 284, 366-371.

37 P. T. Callaghan, Principles of nuclear magnetic resonance microscopy, Clarendon, Oxford, 1991.

38 J. E. Tanner, Use of the stimulated echo in NMR diffusion studies, J. Chem. Phys., 1970, 52, 2523-2526.

39 M. Levitt, Spin Dynamics. Basics of Nuclear Magnetic Resonance, Wiley \& Sons, New York, 2nd edn, 2008.

40 http://www.nmrdb.org/13c/index.shtml?v=v2.95.0.

41 N. Yaghini, L. Nordstierna and A. Martinelli, Effect of water on transport properties of protic and aprotic imidazolium ionic liquids - an analysis of self-diffusivity, conductivity, and proton exchange mechanism, Phys. Chem. Chem. Phys., 2014, 16, 9266-9275.

42 A. Filippov, N. Azancheev, M. Taher, F. U. Shah, P. Rabet, S. Glavatskih and O. N. Antzutkin, Self-diffusion and interactions in mixtures of imidazolium bis(mandelato)borate ionic liquids with poly(ethylene glycol): ${ }^{1} \mathrm{H}$ NMR study, Magn. Reson. Chem., 2015, 53, 493-497.

43 O. Hollóczki, Uranyl(vi) complexes in and from imidazolium acetate ionic liquids: carbenes versus acetates? Inorg. Chem., 2013, 53, 835-846.

44 S. Wellens, N. R. Brooks, B. Thijs, L. van Meervelt and K. Binnemans, Carbene formation upon reactive dissolution 
of metal oxides in imidazolium ionic liquids, Dalton Trans., 2014, 43, 3443-3452.

45 Z. Kelemen, B. Péter-Szabó, E. Székely, O. Hollóczki, D. S. Firaha, B. Kirchner, J. Nagy and L. Nyulászi, An abnormal N-heterocyclic carbene-carbon dioxide adduct from imidazolium acetate ionic liquids: the importance of basicity, Chem. - Eur. J., 2014, 20, 13002-13008.

46 H. Rodríguez, G. Gurau, J. D. Holbrey and R. D. Rogers, Reaction of elemental chalcogens with imidazolium acetates to yield imidazole-2-chalcogenones: direct evidence for ionic liquids as proto-carbenes, Chem. Commun., 2011, 47, 3222-3224.

47 S. Gehrke, W. Reckien, I. Palazzo, T. Welton and O. Hollóczki, On the carbene-like reactions of imidazolium acetate ionic liquids: can theory and experiments agree? Eur. J. Org. Chem., 2019, 504-511.

48 G. A. C. M. Spierings, Wet chemical etching of silicate glasses in hydrofluoric acid based solutions, J. Mater. Sci., 1993, 28, 6261-6273. 